\section{Arkivoc

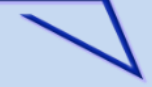

Archive for

Organic Chemistry
The Free Internet Journal

for Organic Chemistry
Paper

Arkivoc 2019, part vi, 0-0

\title{
Synthesis and bioactivity of
}

\section{novel 2-(1,2-benzisothiazol-3-yloxy)-N-(1-aryl-3-cyanopyrazol-5-yl) acetamides}

\author{
Peng Yu*, Xi Li, Xiao Zhang, and Yanhua Xu \\ College of Environmental Science and Engineering, Nanjing Tech University, Nanjing \\ Email:yspong@163.com
}

Received 10-17-2019

Accepted 12-16-2019

Published on line $01-24-2020$

\section{Abstract}

Nine novel types of 2-(1,2-benzisothiazol-3-yloxy)- $N$-(1-aryl-3-cyanopyrazol-5-yl)-acetamides were synthesized, and their inhibition effects on selected bacteria (heterotrophic bacteria) and algae (marine chlorella) were evaluated. Results showed that 2-(1,2-benzisothiazol-3-yloxy)-N-(3-cyano-1-(2,4dimethylphenyl)pyrazol-5-yl) acetamide achieved the highest yield of $81 \%$ with good bioactivity against heterotrophic bacteria and marine chlorella.
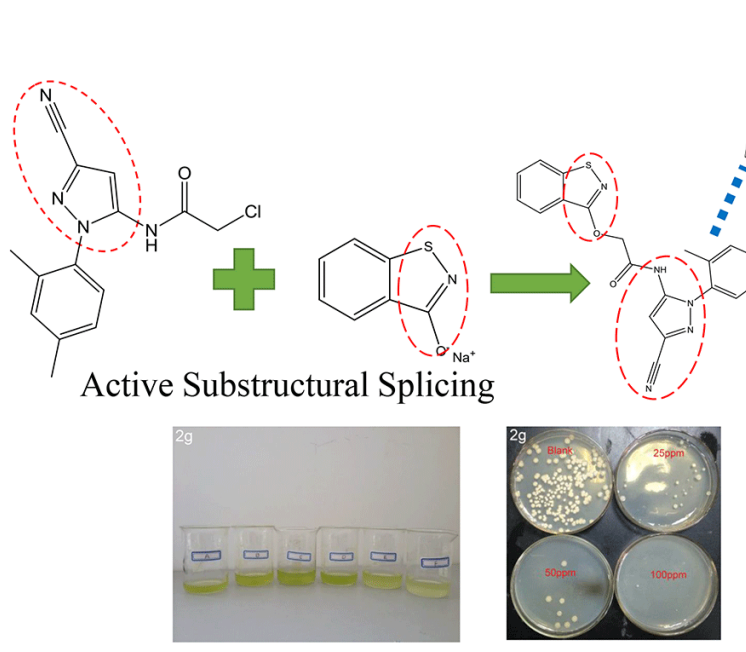

Marine Chlorella

Heterotrophic Bacteria

Bioactivity Inhibition

Keywords: $N$-Aryl pyrazole, isothiazolinone, synthesis, bioactivity 


\section{Introduction}

Over the past years, $\mathrm{N}$-arylpyrazole-containing heterocyclic scaffolds have been widely studied in many fields because of its diverse biological activities, such as antimicrobial, insecticide, and herbicidal. ${ }^{1-5}$ Meanwhile, isothiazolone has attracted considerable interest because of its antimicrobial activity against bacteria and fungi, ${ }^{6,7}$ Derivatives of isothiazolone also exhibit excellent performance as antibacterial and antifungal agents and enzyme inhibitors. ${ }^{8-13}$ Multifunctional water treatment agents are currently a hot research topic. ${ }^{14-15}$ To give the target compound excellent biological activity and a broad spectrum of activity, we introduced an $\mathrm{N}$ arylpyrazole structure, following the principle of sub-structure group splicing. ${ }^{16-18}$

\section{Results and Discussion}

Synthesis. In connection with our research on the synthesis of novel 2-(1,2-benzisothiazol-3-yloxy)- $N$-(3cyano-1-(substituted-phenyl)pyrazol-5-yl) acetamides aimed at achieving multi-functional antifouling agents, we describe the synthesis, one crystal structure, and potential antimicrobial activity of these compounds. Results showed them to exhibit a broad spectrum of activity against heterotrophic bacteria and chlorella. The synthesis route is shown in Scheme 1: 2-(1,2-benzisothiazol-3-yloxy)- $N$-(3-cyano-1-(substitutedphenyl)pyrazole-5-yl) acetamides (8a-8i) were prepared by the reaction of 3-cyano-5-amino-1-(substitutedphenyl)pyrazole chloroacetamides (5) and 1,2-benzisothiazol-3-one sodium salt (7).

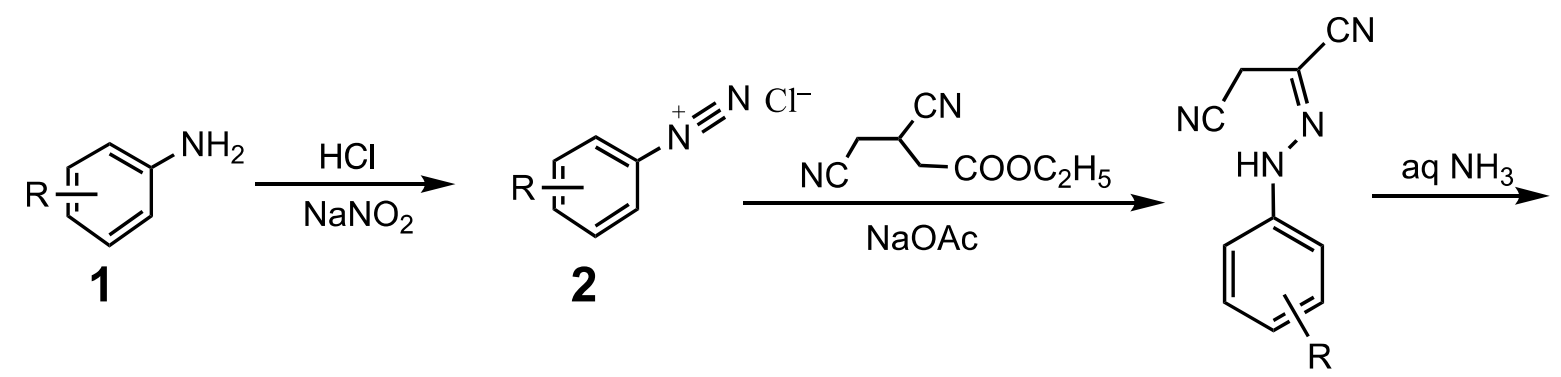

3
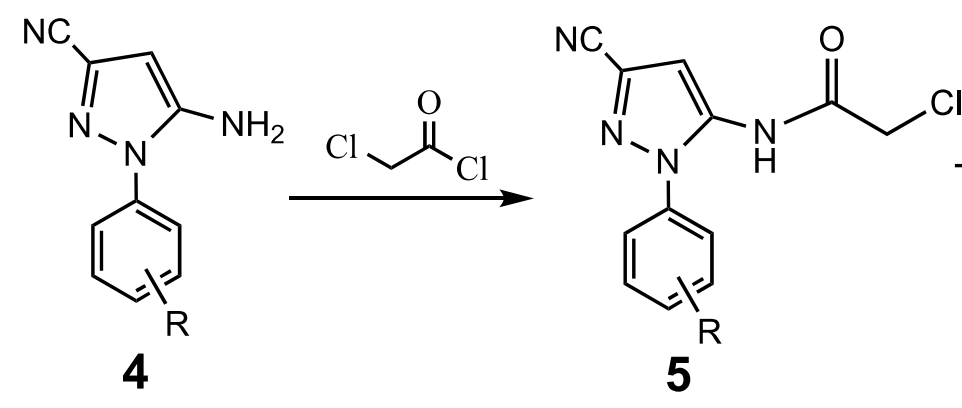<smiles>O=c1[nH]sc2ccccc12</smiles>

6

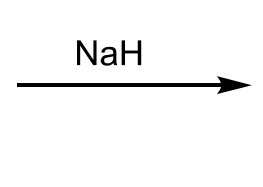<smiles>[NH3+][NH2+]c1nsc2ccccc12</smiles>

8
$(8 a-8 i)$

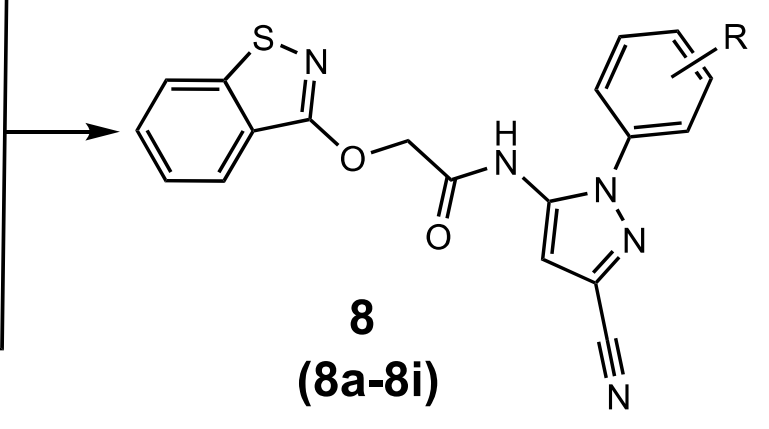

Scheme 1. 
Table 1. Synthesis of compounds $\mathbf{8 a - 8} \mathbf{i}$

\begin{tabular}{ccccc}
\hline Compound & $\mathrm{R}$ & Time $(\mathrm{h})$ & Yield $(\%)$ & Melting Point $\left({ }^{\circ} \mathrm{C}\right)$ \\
\hline $\mathbf{8 a}$ & $\mathrm{H}$ & 5 & 72 & $159-161$ \\
$\mathbf{8 b}$ & 2-methoxy & 5 & 70 & $158-160$ \\
$\mathbf{8 c}$ & 4-methoxy & 5 & 68 & $178-179$ \\
$\mathbf{8 d}$ & 4-methyl & 5 & 69 & $186-188$ \\
$\mathbf{8 e}$ & 4-chloro & 5 & 59 & $188-190$ \\
$\mathbf{8 f}$ & 4-bromine & 5 & 54 & $199-201$ \\
$\mathbf{8 g}$ & 2,4-dimethyl & 5 & 81 & $142-144$ \\
$\mathbf{8 h}$ & 2,6-dichloro & 5 & 72 & $196-198$ \\
$\mathbf{8 i}$ & 2,4,6-trichloro & 5 & 62 & $182-184$ \\
\hline
\end{tabular}

Compound $\mathbf{8 g}$ was subjected to single crystal X-ray crystallography, and intensity data were measured using an Enraf-Nonius CAD4 four-circle diffractometer. Under the condition of 293(2)K, the MoK $\alpha$ ray $(\lambda=0.71073 \AA)$ monochromated with a graphite monochromator was irradiated in $\omega / 2 \theta$ scanning modes and XSCANS. Diffraction data were collected between $2.2^{\circ}<\theta<25.4^{\circ}$, and 3656 diffraction points were collected and reduced, including 3063 independent diffraction points, 1534 strong points $(I>2 \sigma)$, and $R$ (int)=0.056 after absorption correction, orthogonal crystal system, space group Pbca, refinement result $R=0.0789, w R=0.1341$ $(w=1 /[\sigma 2$ (Fo 2) $+(0.1000 P) 2+0.0000 P], P=(F o 2+8 f c 2) / 3), S=1.008$. The highest peak of residual electron density is 0.166 e/Å3, and the lowest valley is -0.218 e/Å3. SHELXS-97 program was used to identify the rough structure by a direct method, and the data were refined by the SHEXLX-97 full matrix least squares method (Fig. 2), with all non-hydrogen atoms anisotropically corrected. All hydrogen atoms underwent theoretical hydrogenation. ${ }^{19,20}$

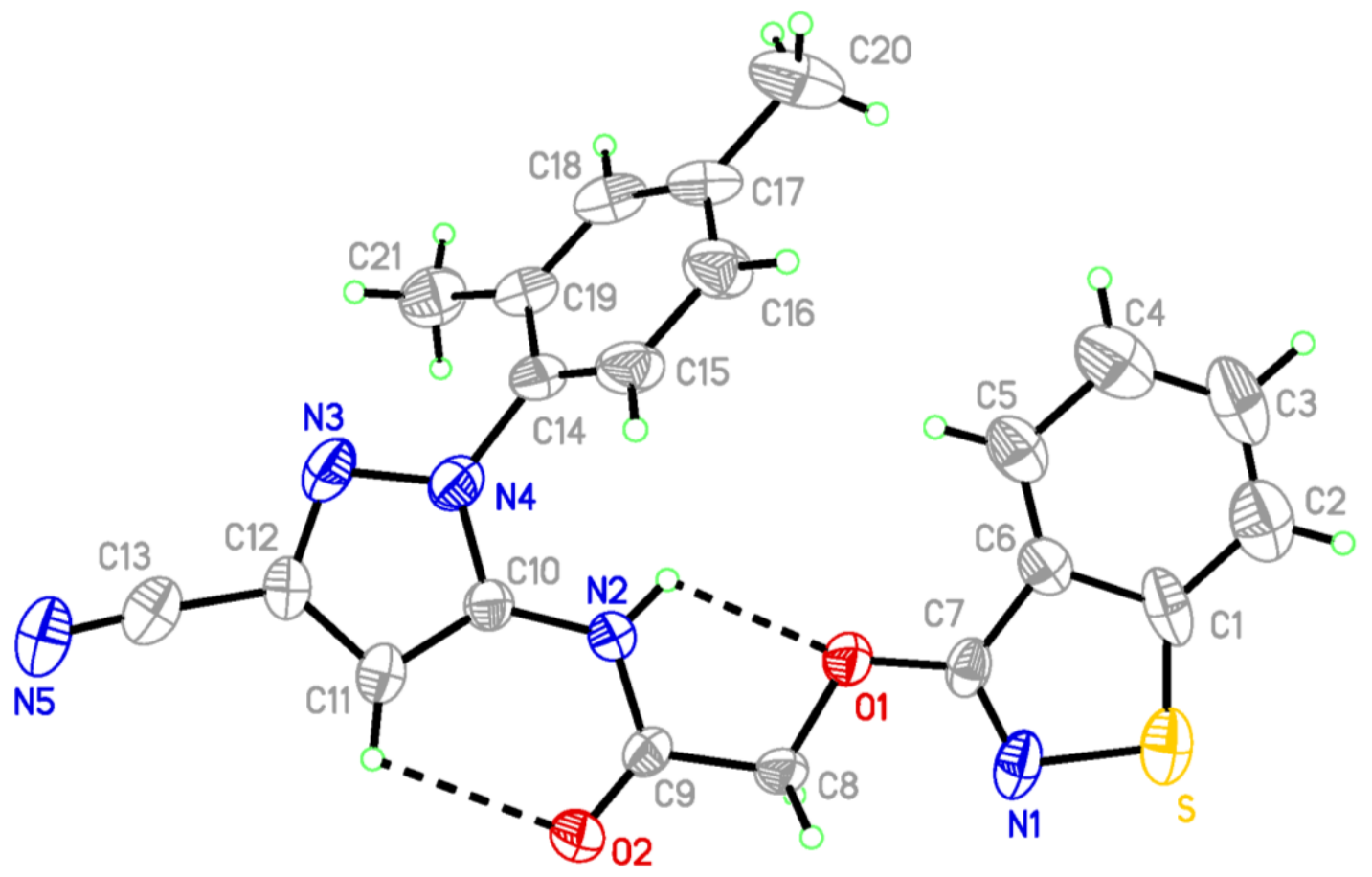

Figure 1. ORTEP diagram of $\mathbf{8 g}$. thermal ellipsoids are shown at the $30 \%$ probability level. 


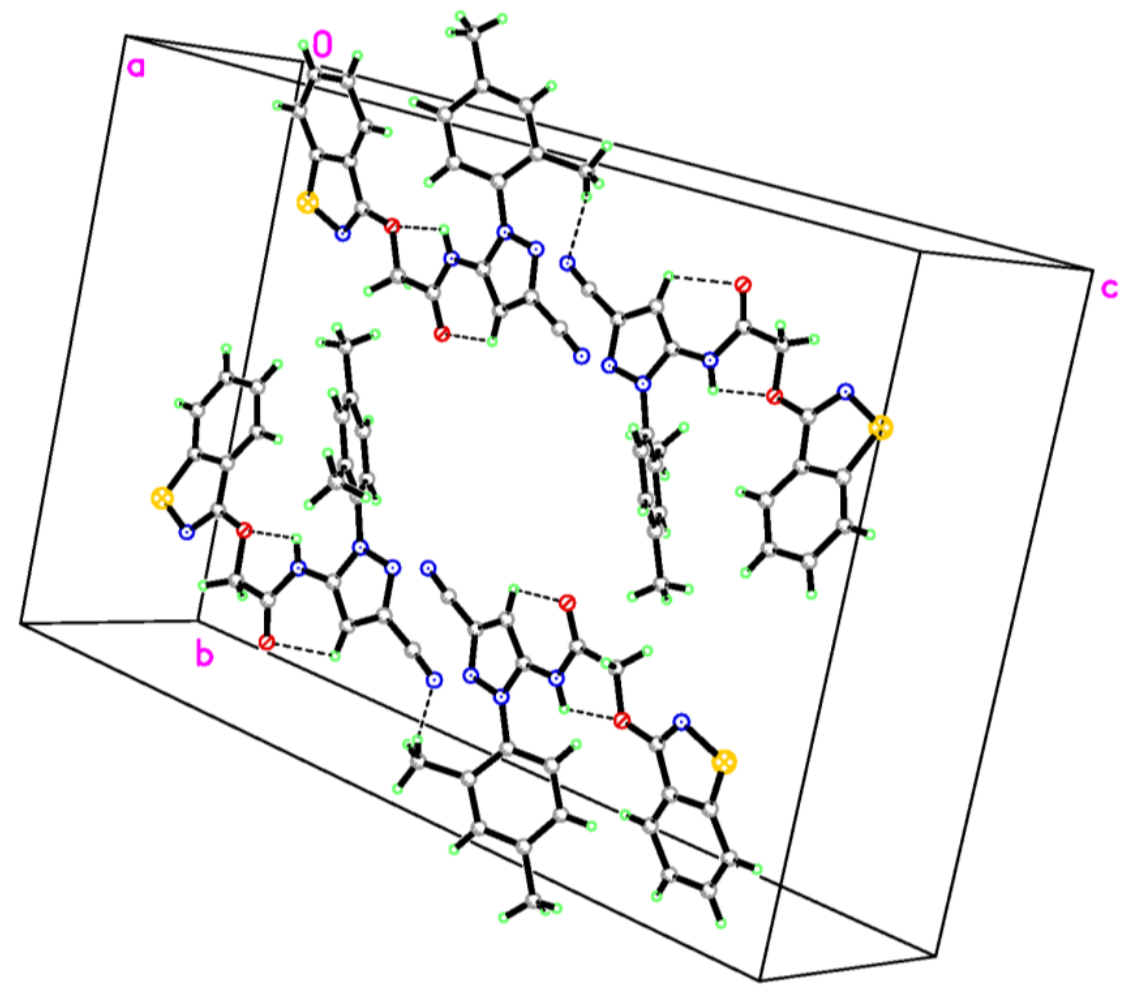

Figure 2. Crystal packing of $\mathbf{8 g}$.

Table 2. Crystal data and structure refinement of compound $\mathbf{8 g}$

\begin{tabular}{cc}
\hline Empirical formula & $\mathrm{C}_{21} \mathrm{H}_{17} \mathrm{~N}_{5} \mathrm{O}_{2} \mathrm{~S}$ \\
\hline CCDC number & 980925 \\
Formula weight $(\mathrm{g} / \mathrm{mol})$ & 403.46 \\
Crystal size $(\mathrm{mm})$ & $0.30 \times 0.20 \times 0.10$ \\
Crystal system & Orthorhombic \\
Space group & $\mathrm{Pbca}$ \\
$\mathrm{a}(\AA)$ & $7.1230(14)$ \\
$\mathrm{b}(\AA)$ & $18.236(4)$ \\
$\mathrm{c}(\AA)$ & $30.579(6)$ \\
$\mathrm{V}(\AA \AA 3)$ & $3972.1(14)$ \\
$\mathrm{Z}$ & 8 \\
Crystal density & 1.349 \\
$\lambda($ MoKa) $(\AA)$ & 0.71073 \\
$\mathrm{~F}(000)$ & 1680 \\
$\mathrm{~h}, \mathrm{k}$ and I ranges & $2.2^{\circ}$ to $25.4^{\circ}$ \\
Reflections measured & $0 \leq \mathrm{h} \leq 8,0 \leq \mathrm{k} \leq 21,0 \leq \mathrm{I} \leq 36$ \\
Independent reflections & 3656 \\
Observed reflection $(\mathrm{I}>2 \sigma(\mathrm{I}))$ & 3603 \\
Rint & 1534 \\
Final $\mathrm{R}^{*}$ indices $(\mathrm{I}>2 \sigma(\mathrm{I}))$ & 0.056 \\
Goodness-of-fit & $\mathrm{R}=0.079, \mathrm{wR}=0.165$ \\
\hline
\end{tabular}


Table 3. Selected Bond Distances $(\AA)$ and Bond Angles $\left({ }^{\circ}\right)$ of $\mathbf{8 g}$

\begin{tabular}{|c|c|c|c|c|c|}
\hline Bond & Dist. & Bond & Dist. & Bond & Dist. \\
\hline $\mathrm{S}-\mathrm{C} 1$ & $1.646(5)$ & $\mathrm{C} 2-\mathrm{C} 3$ & $1.376(7)$ & C12-C13 & $1.436(6)$ \\
\hline S-N1 & $1.671(3)$ & N4-N3 & $1.361(4)$ & C14-C15 & $1.355(5)$ \\
\hline $\mathrm{O} 1-\mathrm{C7}$ & $1.343(4)$ & N4-C10 & $1.371(5)$ & C14-C19 & $1.390(5)$ \\
\hline O1-C8 & $1.448(4)$ & N4-C14 & $1.441(5)$ & C15-C16 & $1.391(5)$ \\
\hline $\mathrm{N} 1-\mathrm{C} 7$ & $1.287(5)$ & C3-C4 & $1.418(7)$ & $\mathrm{C} 16-\mathrm{C} 17$ & $1.385(5)$ \\
\hline $\mathrm{C} 1-\mathrm{C} 2$ & $1.398(6)$ & N5-C13 & $1.148(5)$ & C17-C18 & $1.373(6)$ \\
\hline C1-C6 & $1.445(5)$ & $\mathrm{C} 4-\mathrm{C} 5$ & $1.411(5)$ & C17-C20 & $1.512(6)$ \\
\hline $\mathrm{N} 2-\mathrm{C} 9$ & $1.368(4)$ & C10-C11 & $1.365(5)$ & C18-C19 & $1.395(5)$ \\
\hline N2-C10 & $1.388(4)$ & C11-C12 & $1.370(5)$ & C19-C21 & $1.493(5)$ \\
\hline $\mathrm{C} 5-\mathrm{C} 6$ & $1.364(5)$ & $\mathrm{C} 6-\mathrm{C} 7$ & $1.419(5)$ & $\mathrm{C} 8-\mathrm{C} 9$ & $1.498(5)$ \\
\hline $\mathrm{O} 2-\mathrm{CP}$ & $1.212(4)$ & C12-N3 & $1.334(5)$ & & \\
\hline Angle & $\left({ }^{\circ}\right)$ & Angle & $\left({ }^{\circ}\right)$ & Angle & $\left({ }^{\circ}\right)$ \\
\hline C1-S-N1 & $93.9(2)$ & C5-C6-C1 & $124.3(4)$ & C11-C12-C13 & $127.6(4)$ \\
\hline C7-01-C8 & $114.8(3)$ & C7-C6-C1 & $105.5(4)$ & N5-C13-C12 & $176.1(5)$ \\
\hline C7-N1-S & $110.6(3)$ & N1-C7-O1 & $122.3(4)$ & C12-N3-N4 & $103.0(3)$ \\
\hline C2-C1-C6 & $115.8(5)$ & N1-C7-C6 & $118.0(4)$ & C15-C14-C19 & $122.2(4)$ \\
\hline C2-C1-S & $132.3(5)$ & O1-C7-C6 & $119.6(4)$ & C15-C14-N4 & $117.6(4)$ \\
\hline C6-C1-S & $111.9(3)$ & O1-C8-C9 & $110.1(3)$ & C19-C14-N4 & $120.3(4)$ \\
\hline C9-N2-C10 & $122.8(3)$ & O2-C9-N2 & $123.6(3)$ & C14-C15-C16 & $120.6(4)$ \\
\hline C3-C2-C1 & $120.9(6)$ & $\mathrm{O} 2-\mathrm{C} 9-\mathrm{C} 8$ & $120.1(4)$ & C17-C16-C15 & $119.4(4)$ \\
\hline N3-N4-C10 & $111.5(3)$ & $\mathrm{N} 2-\mathrm{C} 9-\mathrm{C} 8$ & $116.2(3)$ & C18-C17-C16 & $118.4(4)$ \\
\hline N3-N4-C14 & $121.5(3)$ & C11-C10-N4 & $107.0(3)$ & C18-C17-C20 & $121.4(4)$ \\
\hline C10-N4-C14 & 127.1(3) & C11-C10-N2 & $132.8(4)$ & C16-C17-C20 & $120.2(5)$ \\
\hline C2-C3-C4 & $121.9(5)$ & N4-C10-N2 & $120.2(3)$ & C17-C18-C19 & $123.5(4)$ \\
\hline C5-C4-C3 & $118.8(5)$ & C10-C11-C12 & $104.5(4)$ & C14-C19-C18 & $115.8(4)$ \\
\hline C6-C5-C4 & $118.1(5)$ & N3-C12-C11 & $114.0(4)$ & C14-C19-C21 & $124.3(4)$ \\
\hline C5-C6-C7 & $130.1(4)$ & N3-C12-C13 & $118.4(4)$ & C18-C19-C21 & $119.9(4)$ \\
\hline
\end{tabular}

Table 4. Intramolecular and intermolecular hydrogen bonds of $\mathbf{8 g}$

\begin{tabular}{ccccc}
\hline D-H...A & $d(D-H)$ & $d(H \ldots A)$ & $d(D \ldots A)$ & $\angle D H A$ \\
\hline N2-H2A..01 & 0.86 & 2.18 & $2.604(3)$ & 110 \\
C11-H11A. .02 & 0.93 & 2.45 & $2.869(5)$ & 107 \\
C21-H21C..N5 i & 0.96 & 2.59 & $3.535(5)$ & 168 \\
\hline
\end{tabular}

Symmetry codes: (i) $x-1 / 2,-y+1 / 2,-z$. 
Figs. 1 and 2 correspond to compound $8 \mathrm{~g}$, Tables 2 and 3 provide detailed information on the parameters of the compound and the major bond lengths and bond angles. Table 4 shows the hydrogen bonds in the crystal structure. The C-C bonds have lengths of 1.355(5)-1.512(6) $\AA$, which are typical C-C bond lengths and consistent with the literature. Due to the conjugation effect of the pyrazole ring, the C14-N4 bond is long (1.441(5) $\AA$ ), which is between a standard $\mathrm{C}-\mathrm{N}$ single bond $(1.471 \AA$ ) and a $\mathrm{C}=\mathrm{N}$ double bond $(1.273 \AA$ ). The range of the bond angles between the atoms on the benzene ring is between $115.8(5)^{\circ}$ and $124.3(4)^{\circ}$, but the average value is $120^{\circ}$, indicating that the benzene ring is a stable coplanar six-membered ring. The intermolecular hydrogen bonds $\mathrm{N}$ $\mathrm{H} \cdot \mathrm{O}$ and $\mathrm{C}-\mathrm{H} \cdot \mathrm{O}$ form a twisted five-membered ring and a six-membered ring, respectively, and the intermolecular hydrogen bond $\mathrm{C}-\mathrm{H} \cdot \cdot \mathrm{N}$ causes the molecule to form a multi-layered structure.

Crystallographic data for the structure reported in this paper have been deposited $\mathrm{n}$ the Cambridge data center with deposition number CCDC 980925.

Biological assay: Batch antimicrobial experiments were conducted in six parallel samples, and the average results are listed in Table 6. Heterotrophic bacteria and marine chlorella were selected as typical targets for their normal existence in bodies of water, and are important parameters reflecting water quality. The results showed that the target compounds exert good inhibition against the tested organisms.

Table 6. Comparison of the bioactivity of synthesized compounds 8 against heterotrophic bacteria and marine chlorella

\begin{tabular}{ccccc}
\hline \multirow{2}{*}{ Compound } & \multicolumn{2}{c}{ Inhibition rate of heterotrophic bacteria (\%) } & Inhibition rate of Marine Chlorella (\%) \\
& 100ppm & 50ppm & 25ppm & 60.29 \\
\hline $\mathbf{8 a}$ & 91.3 & 85.3 & 62.1 & 65.81 \\
$\mathbf{8 b}$ & 94.3 & 86.9 & 75.5 & 65.305 \\
$\mathbf{8 c}$ & 94.5 & 90.2 & 81.2 & 67.955 \\
$\mathbf{8 d}$ & 100 & 98.2 & 80.7 & 68.48 \\
$\mathbf{8 e}$ & 98.9 & 88.4 & 58.9 & 72.5 \\
$\mathbf{8 f}$ & 95.2 & 85.2 & 67.8 & 71.02 \\
$\mathbf{8 g}$ & 97.7 & 90.2 & 68.5 & 73.99 \\
$\mathbf{8 h}$ & 96.2 & 81.6 & 64.3 & 74.01 \\
$\mathbf{8 i}$ & 100 & 95.3 & 81.9 & 53.5 \\
\hline
\end{tabular}

\section{Conclusions}

This study demonstrated the synthesis and antimicrobial activities of 2-(benzisothiazol-3-yloxy)-N-(3-cyano-1aryl-5-yl) acetamides. Bioactivity testing showed that the compounds exert satisfactory inhibition effects against heterotrophic bacteria and marine chlorella, with rates more than $58.9 \%$ and $60.29 \%$ for heterotrophic bacteria and marine chlorella at a dosage of $25 \mathrm{ppm}$, respectively. The highest yield obtained was $81 \%$, indicating that compounds with an aryl-pyrazole and a benzisothiazolone spliced together have high biological activities and low toxicities and represent a new direction for further structural optimization and biological studies on such synthetic acetamides. 


\section{Experimental Section}

General. All chemicals used in this study were commericallly available. Melting points were recorded on a X-4 binocular spectra melting apparatus. IR spectra in $\mathrm{KBr}$ were recorded on a PerkinElmer PE-683 IR spectrometer. The ${ }^{1} \mathrm{H}$ NMR spectra were determined using TMS as an internal reference with a Avance Bruker-500 instrument operating at $500 \mathrm{MHz}$ or an Avance Bruker-300 instrument operating at $400 \mathrm{MHz}$. Elemental analyses were performed by an Elementer Vario EL III elementary analysis instrument. Compounds were synthesized in accordance the method described in our previous work. ${ }^{21}$

Benzisothiazolinone sodium salt. A mixture of benzisothiazol-3(8H)-one (6) (0.01 mol) and $\mathrm{NaH}(0.011 \mathrm{~mol})$ in $\mathrm{MeOH}(40 \mathrm{~mL})$ was stirred and heated at refluxed for $2 \mathrm{~h}$. The remaining $\mathrm{MeOH}$ was evaporated under negative pressure. The crude product was washed with $\mathrm{MeOH}$ and dried to obtain benzisothiazolin-3-one sodium salt (7) as a white solid, $\mathrm{mp}>300^{\circ} \mathrm{C}$.

3-Cyano-5-amino-1-(substituted-phenyl)pyrazoles. Ethyl 2,3-dicyanopropanoate (0.01 mol), NaOAc (0.03 mol), and $\mathrm{EtOH}(50 \mathrm{~mL}$ ) were mixed in a $150 \mathrm{~mL}$ round-bottomed flask. Then, the mixture was added to a substituted aniline diazonium salt within $20 \mathrm{~min}$ and stirred at $5{ }^{\circ} \mathrm{C}$ for $8 \mathrm{~h}$, after which, the mixture was extracted with $\mathrm{CH}_{2} \mathrm{Cl}_{2}(3 \times 30 \mathrm{~mL})$. Ammonia was then added to the combined organic extracts to adjust the $\mathrm{pH}$ to 9-10. After addition, the mixture was stirred at $\mathrm{rt}$ for $3 \mathrm{~h}$. The organic phase was washed and then concentrated. After cooling, the crude product precipitated, was filtered off, washed with $\mathrm{EtOH}$, dried, and then recrystallized from toluene to afford the 3-cyano-5-amino-1-(substituted-phenyl)pyrazole (4).

3-Cyano-5-amino-1-(substituted-phenyl)pyrazole chloroacetamide. Chloroacetyl chloride (0.015 mol) was added dropwise to a $\mathrm{CH}_{2} \mathrm{Cl}_{2}$ solution of 3-cyano-5-amino-1-(substituted-phenyl)pyrazole (0.01 mol in $\left.30 \mathrm{~mL}\right)$ at $0-5{ }^{\circ} \mathrm{C}$, and the mixture obtained was stirred at $\mathrm{rt}$ for $8 \mathrm{~h}$ with $\mathrm{Et}_{3} \mathrm{~N}(0.015 \mathrm{~mol})$ as an acid acceptor. After the reaction, the remaining $\mathrm{CH}_{2} \mathrm{Cl}_{2}$ was evaporated under reduced pressure to obtain crude 3-cyano-5-amino-1(substituted-phenyl)pyrazole chloroacetamide (5).

Synthesis of 2-(1,2-benzisothiazol-3-yloxy)-N-(3-cyano-1-(substituted-phenyl)pyrazol-5-yl) acetamides (2). With $\mathrm{KI}$ as a catalyst, a benzisothiazolone sodium salt (7) (0.006 mol) was reacted with a solution of 3-cyano-5-amino1-(substituted-phenyl)pyrazole chloroacetamide in DMF $(40 \mathrm{~mL})$, at $100{ }^{\circ} \mathrm{C}$ for $4 \mathrm{~h}$. The solutioin was concentrated and poured into $\mathrm{H}_{2} \mathrm{O}$, and a solid was precipitated, filtered off, washed, and dried. The product (8ai) was purified by column chromatography.

2-(1,2-Benzisothiazol-3-yloxy)- $\mathbf{N}$-(3-cyano-1-phenylpyrazol-5-yl) acetamide (8a). ${ }^{1} \mathrm{H}$ NMR (CDCl, $\left.500 \mathrm{MHz}\right) \delta$ : $5.15\left(\mathrm{~s}, 2 \mathrm{H}, \mathrm{CH}_{2}\right), 7.15(\mathrm{~s}, 1 \mathrm{H}, \mathrm{C}-\mathrm{H}), 7.08 \sim 7.88(\mathrm{~m}, 9 \mathrm{H}, \mathrm{Ar}-\mathrm{H}), 8.60(\mathrm{~s}, 1 \mathrm{H}, \mathrm{N}-\mathrm{H}) ; \mathrm{IR}(\mathrm{KBr}) \mathrm{v}: 3177(\mathrm{~N}-\mathrm{H}), 3058(\mathrm{C}-\mathrm{H})$, 2246(C $\equiv N), 1724(\mathrm{C}=\mathrm{O}), 1596(\mathrm{C}=\mathrm{N}), 1546(\mathrm{C}=\mathrm{C}), 1262(\mathrm{C}-\mathrm{N}), 645(\mathrm{C}-\mathrm{S}) \mathrm{cm}^{-1}$. Anal. Calcd. (\%) for $\mathrm{C}_{19} \mathrm{H}_{13} \mathrm{~N}_{5} \mathrm{O}_{2} \mathrm{~S}: \mathrm{C}$, 60.79; H, 3.49; N, 18.66. Found (\%): C, 61.01; H, 3.35; N, 18.68 .

2-(1,2-Benzisothiazol-3-yloxy)- $\mathbf{N}$-(3-cyano-1-(2-methoxyphenyl)pyrazol-5-yl) acetamide (8b). ${ }^{1} \mathrm{H} \mathrm{NMR}^{\left(C D C l_{3}\right.}$, $500 \mathrm{MHz}) \delta: 3.84\left(\mathrm{~s}, 3 \mathrm{H}, \mathrm{CH}_{3} \mathrm{O}\right), 5.17\left(\mathrm{~s}, 2 \mathrm{H}, \mathrm{CH}_{2}\right), 7.14(\mathrm{~s}, 1 \mathrm{H}, \mathrm{C}-\mathrm{H}), 6.78 \sim 7.87(\mathrm{~m}, 8 \mathrm{H}, \mathrm{Ar}-\mathrm{H}), 8.31(\mathrm{~s}, 1 \mathrm{H}, \mathrm{N}-\mathrm{H}) ; \mathrm{IR}$ $(\mathrm{KBr})$ v: 3295(N-H), 3170(C-H), 2241(C=N), 1713(C=O), 1596(C=N), 1557(C=C), 1321(C-N), 647(C-S) cm $\mathrm{cm}^{-1}$ Anal. Calcd. (\%) for $\mathrm{C}_{20} \mathrm{H}_{15} \mathrm{~N}_{5} \mathrm{O}_{3} \mathrm{~S}: \mathrm{C}, 59.25 ; \mathrm{H}, 3.73 ; \mathrm{N}, 17.27$. Found (\%): C, 59.28; $\mathrm{H}, 3.61 ; \mathrm{N}, 17.29$.

2-(1,2-Benzisothiazol-3-yloxy)-N-(3-cyano-1-(4-methoxyphenyl) pyrazol-5-yl) acetamide (8c). ${ }^{1} \mathrm{H} \mathrm{NMR} \mathrm{(CDCl}$, $500 \mathrm{MHz}) \delta: 3.85\left(\mathrm{~s}, 3 \mathrm{H}, \mathrm{CH}_{3} \mathrm{O}\right), 5.14\left(\mathrm{~s}, 2 \mathrm{H}, \mathrm{CH}_{2}\right), 6.99(\mathrm{~s}, 1 \mathrm{H}, \mathrm{C}-\mathrm{H}), 7.01 \sim 7.82(\mathrm{~m}, 8 \mathrm{H}, \mathrm{Ar}-\mathrm{H}), 8.34(\mathrm{~s}, 1 \mathrm{H}, \mathrm{N}-$ $\mathrm{H})$; IR ( $\mathrm{KBr})$ v: 3383(N-H), 3167(C-H), 2241(C=N), 1717(C=O), 1597(C=N), 1557(C=C), 1323(C-N), 1225(C-F), 647(C-S) cm $\mathrm{cm}^{-1}$. Anal. Calcd. (\%) for $\mathrm{C}_{20} \mathrm{H}_{15} \mathrm{~N}_{5} \mathrm{O}_{3} \mathrm{~S}$ : C, 59.25; H, 3.73; N, 17.27. Found (\%): C, 59.20; $\mathrm{H}, 3.62 ; \mathrm{N}, 17.35$. 2-(1,2-Benzisothiazol-3-yloxy)-N-(3-cyano-1-(4-methylphenyl)pyrazol-5-yl) acetamide (8d). ${ }^{1} \mathrm{H} \mathrm{NMR}\left(\mathrm{CDCl}_{3}, 500\right.$ 
$\mathrm{MHz})$ \&: $2.39\left(\mathrm{~s}, 3 \mathrm{H}, \mathrm{CH}_{3}\right), 5.15\left(\mathrm{~s}, 2 \mathrm{H}, \mathrm{CH}_{2}\right), 7.18(\mathrm{~s}, 1 \mathrm{H}, \mathrm{C}-\mathrm{H}), 6.86 \sim 7.88(\mathrm{~m}, 8 \mathrm{H}, \mathrm{Ar}-\mathrm{H}), 8.51(\mathrm{~s}, 1 \mathrm{H}, \mathrm{N}-\mathrm{H}) ; \mathrm{IR}$ $(\mathrm{KBr})$ v: 3401(N-H), 3171(C-H), 2241(C=N), 1725(C=O), 1589(C=N), 1556(C=C), 1324(C-N), 646(C-S) $\mathrm{cm}^{-1}$. Anal. Calcd. (\%) for $\mathrm{C}_{20} \mathrm{H}_{15} \mathrm{~N}_{5} \mathrm{O}_{2} \mathrm{~S}$ : C, 61.68; $\mathrm{H}, 3.88 ; \mathrm{N}, 17.98$. Found (\%): $\mathrm{C}, 61.59 ; \mathrm{H}, 3.77 ; \mathrm{N}, 17.83$.

2-(1,2-Benzisothiazol-3-yloxy)- $\mathbf{N}$-(3-cyano-1-(4-chlorophenyl) pyrazol-5-yl) acetamide (8e). ${ }^{1} \mathrm{H} \mathrm{NMR}\left(\mathrm{CDCl}_{3}, 500\right.$ $\mathrm{MHz}) \delta: 5.16\left(\mathrm{~s}, 2 \mathrm{H}, \mathrm{CH}_{2}\right), 7.18(\mathrm{~s}, 1 \mathrm{H}, \mathrm{C}-\mathrm{H}), 6.99 \sim 7.84(\mathrm{~m}, 8 \mathrm{H}, \mathrm{Ar}-\mathrm{H}), 8.58(\mathrm{~s}, 1 \mathrm{H}, \mathrm{N}-\mathrm{H}) ; \mathrm{IR}(\mathrm{KBr}) \mathrm{v}: 3386(\mathrm{~N}-\mathrm{H})$, 3078(C-H), 2242(C=N), 1712(C=O), 1589(C=N), 1557(C=C), 1334(C-N), 1120(C-F), 647(C-S) cm ${ }^{-1}$. Anal. Calcd. (\%) for $\mathrm{C}_{19} \mathrm{H}_{12} \mathrm{ClN}_{5} \mathrm{O}_{2} \mathrm{~S}: \mathrm{C}, 55.68 ; \mathrm{H}, 2.95 ; \mathrm{N}, 17.09$. Found (\%): C, 55.75; $\mathrm{H}, 2.90 ; \mathrm{N}, 17.14$.

2-(1,2-Benzisothiazol-3-yloxy)-N-(3-cyano-1-(4-bromophenyl)pyrazol-5-yl)acetamide (8f). ${ }^{1} \mathrm{H} N M R$ (CDCl3, 500 $\mathrm{MHz})$ : $5.15(\mathrm{~s}, 2 \mathrm{H}, \mathrm{CH} 2), 7.19(\mathrm{~s}, 1 \mathrm{H}, \mathrm{C}-\mathrm{H}), 6.98 \sim 7.85(\mathrm{~m}, 8 \mathrm{H}, \mathrm{Ar}-\mathrm{H}), 8.60(\mathrm{~s}, 1 \mathrm{H}, \mathrm{N}-\mathrm{H}) ; \mathrm{IR}(\mathrm{KBr}) \mathrm{v}: 3259(\mathrm{~N}-$ $\mathrm{H}), \quad 3057(\mathrm{C}-\mathrm{H}), \quad 2374(\mathrm{C} \equiv \mathrm{N}), 1674(\mathrm{C}=\mathrm{O}), 1597(\mathrm{C}=\mathrm{N}), 1539(\mathrm{C}=\mathrm{C}), 1290(\mathrm{C}-\mathrm{N}), 1066(\mathrm{C}-\mathrm{Br}), 650(\mathrm{C}-\mathrm{S}) \mathrm{cm}-1$. Anal. Calcd. (\%) for C19H12BrN5O2S: C, 50.23; H, 2.66; N, 15.42. Found (\%): C, 50.34; H, 2.53; N, 15.38.

2-(1,2-Benzisothiazol-3-oxy)- $\mathbf{N}$-(3-cyano-1-(2,4-dimethylphenyl)pyrazole-5-yl) acetamide (8g). ${ }^{1} \mathrm{H} N \mathrm{NM}\left(\mathrm{CDCl}_{3}\right.$, $500 \mathrm{MHz}) \delta: 2.02\left(\mathrm{~s}, 6 \mathrm{H}, \mathrm{CH}_{3}\right), 5.11\left(\mathrm{~s}, 2 \mathrm{H}, \mathrm{CH}_{2}\right), 7.12(\mathrm{~s}, 1 \mathrm{H}, \mathrm{C}-\mathrm{H}), 6.74 \sim 7.85(\mathrm{~m}, 7 \mathrm{H}, \mathrm{Ar}-\mathrm{H}), 8.46(\mathrm{~s}, 1 \mathrm{H}, \mathrm{N}-\mathrm{H})$; IR (KBr) v: 3386(N-H), 3169(C-H), 2243(C=N), 1716(C=O), 1595(C=N), 1558(C=C), 1323(C-N), 648(C-S) cm ${ }^{-1}$. Anal. Calcd. (\%) for $\mathrm{C}_{21} \mathrm{H}_{17} \mathrm{~N}_{5} \mathrm{O}_{2} \mathrm{~S}: \mathrm{C}, 62.52 ; \mathrm{H}, 4.25 ; \mathrm{N}, 17.36$. Found (\%): C, 62.42; $\mathrm{H}, 4.18 ; \mathrm{N}, 17.43$.

2-(1,2-Benzisothiazol-3-yloxy)- $\mathbf{N}$-(3-cyano-1-(2,6-dichlorophenyl)pyrazol-5-yl) acetamide (8h). ${ }^{1} \mathrm{H} \mathrm{NMR}\left(\mathrm{CDCl}_{3}\right.$, $500 \mathrm{MHz}) \delta: 5.16\left(\mathrm{~s}, 2 \mathrm{H}, \mathrm{CH}_{2}\right), 7.22(\mathrm{~s}, 1 \mathrm{H}, \mathrm{C}-\mathrm{H}), 7.11 \sim 7.86(\mathrm{~m}, 7 \mathrm{H}, \mathrm{Ar}-\mathrm{H}), 8.77$ (s, 1H, N-H); IR $(\mathrm{KBr}) v$ : 3202(N-H), 3056(C-H), 2242(C=N), 1689(C=O), 1596(C=N), 1560(C=C), $1259(\mathrm{C}-\mathrm{N}), 1146(\mathrm{C}-\mathrm{Cl}), 649(\mathrm{C}-\mathrm{S}) \mathrm{cm}^{-1}$. Anal. Calcd. (\%) for $\mathrm{C}_{19} \mathrm{H}_{11} \mathrm{C}_{12} \mathrm{~N}_{5} \mathrm{O}_{2} \mathrm{~S}: \mathrm{C}, 51.36 ; \mathrm{H}, 2.50 ; \mathrm{N}, 15.76$. Found (\%): $\mathrm{C}, 51.30 ; \mathrm{H}, 2.54 ; \mathrm{N}, 15.82$.

2-(1,2-Benzisothiazol-3-yloxy)- $\mathrm{N}$-(3-cyano-1-(2,4,6-dichlorophenyl)pyrazol-5-yl) acetamide (8i). ${ }^{1} \mathrm{H} \mathrm{NMR} \mathrm{(CDCl}$, $500 \mathrm{MHz}) \delta: 5.18\left(\mathrm{~s}, 2 \mathrm{H}, \mathrm{CH}_{2}\right), 7.25(\mathrm{~s}, 1 \mathrm{H}, \mathrm{C}-\mathrm{H}), 7.11 \sim 7.86(\mathrm{~m}, 6 \mathrm{H}, \mathrm{Ar}-\mathrm{H}), 8.75(\mathrm{~s}, 1 \mathrm{H}, \mathrm{N}-\mathrm{H}) ; \mathrm{IR}(\mathrm{KBr}) v$ : 3246(N-H), 3078(C-H), 2247(C=N), 1718(C=O), 1654(C=N), 1556(C=C), 1388(C-N), 1168(C-Cl), 651(C-S) cm ${ }^{-1}$. Anal. Calcd. (\%) for $\mathrm{C}_{19} \mathrm{H}_{10} \mathrm{Cl}_{3} \mathrm{~N}_{5} \mathrm{O}_{2} \mathrm{~S}: \mathrm{C}, 47.67 ; \mathrm{H}, 2.11 ; \mathrm{N}, 14.63$. Found (\%): C, 47.72; H, 2.18; N, 14.58.

\section{Acknowledgements}

This work was funded by the National Key R\&D Program of China (Grant No. 2017YFB0602500), Natural Science Foundation of China (21607074), and Natural Science Research Program of Jiangsu (Grant No. 16KJA610002).

\section{References}

1. Cheng, J. L.; Wei, F. L.; Zhu, L.; Zhao J. H.; Zhu, G. N. Chin. J. Org. Chem. 2008, 28, 622.

2. Zhao, Q. Q.; Li, Y. Q.; Xiong L. X.; Wang Q. M. J. Agric. Food Chem. 2010, 58, 4992.

https://doi.org/10.1021/jf1001793

3. Sun, Y. F.; Qiao, H. L.; Ling, Y. ; Yang, S. X.; Rui, C. H.; Pelosi, P.; Yang, X. L. J. Agric. Food. Chem. 2011, 59, 2456.

https://doi.org/10.1021/jf104712c

4. Fu, X. D.; Chen, Y.; Yang, Y.; He, S. Z.; Shen, C.; Wan, R. Chin. J. Org. Chem. 2014, 34, 2090. https://doi.org/10.6023/cjoc201403035

5. Liu, Y. Y.; Shi, H.; Li, Y. F.; Zhu, H. J. J. Heterocycl. Chem. 2010, 47, 897.

https://doi.org/10.1002/jhet.424 
6. Ma D.; Cai, X.; Lin, Q.; Zhang, J.; Mai, W.; Tan, S.; Xue W.; Wu, T. J. Appl. Polym. Sci. 2013, $130,1554$. https://doi.org/10.1002/app.39293

7. Zani F, Vicini P, Incerti M. Eur. J. Med. Chem. 2004, 39, 135. https://doi.org/10.1016/j.ejmech.2003.11.004

8. Zhang, C. Y.; Yu, L. M.; Jiang X. H.; Li, C. C. Environ. Sci. Technol. 2010, 33, 106.

9. Dou, D. F.; Alex D.; Du, B. F. Bioorg. Med. Chem. 2011, 19, 5782. https://doi.org/10.1016/j.bmc.2011.08.029

10. Wang, X. H.; You C. H.; Lin, Q. Chin. J. Struct. Chem. 2013, 32, 1123.

11. Yu, P.; Li, X.; Hu, J.; Xu, Y. H. Chin. J. Struct. Chem. 2014, 33, 1375

12. Ma, X. X.; Zhang H. T.; Zhao, J. Chin. Coatings 2012, 27, 47.

13. Ge, H. H.; Tao, J. T.; Xu, X. M.; Wei C. J.; Meng, X. J. Adv. Mater. Res. 2011, 239, 2261. https://doi.org/10.4028/www.scientific.net/AMR.239-242.2261

14. Viani, F. ; Rossi, B. ; Panzeri, W.; Merlioni, L.; Martorana, A. M. ; Polissi, A. ; Galante, Y. M. Tetrahedron 2017, 73,1745 . https://doi.org/10.1016/j.tet.2017.02.025

15. Jiang, Q.; Xin L. H.; Tian, B. H. Water Resources Protection, 2012, 28, 66.

16. Cheng, Y.; Prud'homme, R. K., Chick, J. ; Rau, D. C. Macromol. 2002, 35, 10155. https://doi.org/10.1021/ma020887e

17. Cheng, Y.; Brown, K. M.; Prud'homme, R. K. Biomacromol. 2002, 3.

18. Kenawy, El-R.; Worley, S. D.; Broughton, R. Biomacromol. 2007, 8, 1359. https://doi.org/10.1021/bm061150q

19. Sheldrick, G. M. Acta Crystallogr. 2008, A64, 112. https://doi.org/10.1107/S0108767307043930

20. Sheldrick, G. M. SHELXL97. University of Götingen, Germany, 1997.

21. P. Yu, J. Pan, and B. M. Liu Compounds having isothiazolinone and $\mathrm{N}$-arylpyrazole structures and preparation and application thereof. CN 109320508A, 2019. 